\section{P441 CONFRONTING THE HIDDEN PUBLIC HEALTH THREAT: EMERGING AND RE-EMERGING SEXUALLY TRANSMITTED INFECTIONS}

S Balaji*. Scientist-B, Indian Council of Medical Research, New Delhi, India

10.1136/sextrans-2021-sti.457

Background Substantial increase in the outbreaks of sexually transmitted infections (STIs) and associated mortalities have raised international concerns. Concurrent with the escalation of established STIs, developing epidemics and outbreaks of newly emerging sexually transmissible pathogens pose serious problems for people and added burden and challenges for public health practitioners and researchers. Importantly, most of the emerging STIs are frequently found among vulnerable groups; such as men having sex with men and HIV patients, which may result in large outbreaks in near future.

Methods In this systematic review, we explored emerging and re-emerging STIs, their current epidemiological status, and future perspectives. This study includes research articles reporting the epidemiology of emerging and re-emerging SITs in the past decade. The keywords 'Emerging and re-merging Sexually transmitted infections' were searched in the PubMed database to collect the articles. After the application of inclusion and exclusion criteria number of articles was reduced from 3278 to 278 .

Results Our study found epidemiological trends and antimicrobial resistance of various emerging and re-emerging bacterial and fungal STIs namely Shigella flexneri, Mycoplasma genitalium, Lymphogranuloma venereum, Lymphogranuloma venereum, Group B Streptococcus, Neisseria meningitides, Methicillin-Resistant Staphylococcus Aureus (MRSA), Hepatitis A \& C, Ebola, Zika, Dengue across the countries.

Conclusion As per the assessment, the incidence of these STIs would continue to arise owing to enhanced human inter-connectedness such as international travel and extensive use of social networks meant for sexual activities. Also, the incidence of oral and anal sex has enormously increased over the past decades, especially, doubled number of anal intercourse has been recorded worldwide in the 21 st century, which is the major contributing factor for the enhanced emerging and reemerging STIs.

\section{P442 CHANGING PATTERNS OF SEXUALLY TRANSMITTED INFECTIONS IN INDIA}

S Aggarwal*. Indian Council of Medical Research, New Delhi, Delhi, India

10.1136/sextrans-2021-sti.458

Background Sexually transmitted infections (STIs) are the most common among notifiable diseases across the countries. About one million STIs are reported every day and more than 376 million cases per year around the globe. Their epidemiological profile varies from country to country and from one region to another within a country, depending on ethnographic, demographic, socioeconomic, and health factors. Thus, the present study was conducted to assess the current trend of occurrence of STIs along with its spectrum of aetiology in India.

Methods This systematic review included research articles addressing the prevalence of various STIs related publications from India between 2000 to 2020. For the article search, PubMed and google scholar were used. The keywords
'Sexually transmitted infections' and 'India' were searched in the PubMed database.

Results This exercise provided us with a large number of articles i.e. 1658, after applying various inclusion and exclusion criteria about 136 articles fit into the criteria. A random search was also done with google scholar, where 16 new relevant articles were found that were not indexed in PubMed. All of them were discussed in relevance to their subjects in the current review. All articles were reviewed thoroughly for the aetiology and occurrence of STIs, then data were extracted from the paper for analysis.

Conclusion The results of the present study clearly indicated the changing trend of STIs in India from bacterial to virus. This necessitates the need for estimation of the current prevalence of STI diseases at the national level in various populations, subpopulation group,s and different geographical areas.

\section{P443 TRANSITIONING TO TELEHEALTH PREP DELIVERY DURING THE COVID-19 PANDEMIC: UPTAKE AND PATIENT PERCEPTIONS}

\begin{abstract}
${ }^{1,2}{ }^{A}$ Riba* ${ }^{*},{ }^{1,3} \mathrm{O}$ Obafemi, ${ }^{1} \mathrm{~J}$ Weise, ${ }^{1} \mathrm{~N}$ Gibson, ${ }^{1,4} \mathrm{~K}$ Wendel. ${ }^{1}$ Denver Prevention Training Center, Denver, USA; ${ }^{2}$ The University of Colorado Anschutz, USA; ${ }^{3}$ Denver Public Health, USA; ${ }^{4}$ Denver Health Infectious Disease Division, USA
\end{abstract}

\subsection{6/sextrans-2021-sti.459}

Background As HIV rates have stagnated across the United States, the rate of new HIV infections in Colorado increased by $1.8 \%$ from 2014-2019. Increasing pre-exposure prophylaxis (PrEP) uptake and retention is an important component of the United States' Ending the HIV Epidemic campaign to address these rates. In 2020, the COVID-19 pandemic significantly limited in-person appointments to the Denver Public Health (DPH) STI Clinic and threatened to derail the clinics PrEP services.

Approach In March 2020, in response to the COVID-19 pandemic and the resultant mandatory stay at home order, the DPH STI Clinic rapidly converted in-clinic PrEP care to a telehealth delivery system. Patients were evaluated for PrEP services via telephone or web-based video platform. In-clinic lab only appointments were completed prior to release of mailed or in-person pick-up PrEP prescriptions. To evaluate patient perceptions, a patient satisfaction survey was electronically delivered to 212 patients receiving telehealth PrEP start visits in the 6 months after implementation.

Outcomes/Impact The DPH STI Clinic was able to maintain 93\% of the monthly PrEP start visit volume (57 PrEP start visits/month average) in the six months after implementing telehealth services compared to the six months prior (61 PrEP start visits/month average). Fourteen percent (30/212) of patients receiving the patient satisfaction survey completed the survey. Greater than 90\% (21/23) of responding patients agreed that telehealth appointments and lab only visits were easy to schedule and complete. Patients preferred telehealth PrEP visits $(92 \% ; 22 / 24)$ to traditional in-clinic visits.

Innovation Telehealth is a rapidly emerging care delivery model that has revolutionized HIV prevention services. Use of telehealth PrEP visits allowed continued support of PrEP initiation during COVID-19 and was well received by patients compared to traditional in-clinic visits. 